\title{
A novel approach to modeling tissue-level activity of cortisol levels according to the theory of Endobiogeny, applied to chronic heart failure
}

\author{
Kamyar M. Hedayat ${ }^{1}$, Jean-Claude Lapraz ${ }^{2}$, Benjamin M. Schuff ${ }^{3}$, Tiffany Barsotti ${ }^{4}$, \\ Shahrokh Golshan ${ }^{5}$, Suzi Hong ${ }^{6}$, Barry H. Greenberg ${ }^{7}$, Paul J. Mills ${ }^{8}$ \\ ${ }^{1,2}$ Systems Biology Research Group, Chicago, USA \\ ${ }^{3}$ National University of Natural Medicine, Portland, Oregon, USA \\ ${ }^{3}$ Whole Systems Healthcare, Portland, Oregon, USA \\ ${ }^{4} \mathrm{Heal}$ and Thrive, Encinitas, USA \\ $5,6,8$ Department of Psychiatry, University of California San Diego, San Diego, USA \\ ${ }^{7}$ Department of Medicine, University of California, San Diego, USA \\ ${ }^{8}$ Department of Family and Preventative Medicine, University of California, San Diego, USA \\ ${ }^{3}$ Corresponding author \\ E-mail: ${ }^{1}$ acquamarine@mac.com, ${ }^{2}$ jeanclaudlapraz@icloud.com, ${ }^{3}$ bschuff1@gmail.com, \\ ${ }^{4}$ tiffany@healandthrive.com, ${ }^{5}$ sgolshan@ucsd.edu, ${ }^{6}$ slhong@ucsd.edu, ${ }^{7}$ bgreenberg@ucsd.edu, \\ 8pmills@ucsd.edu
}

Received 6 May 2018; received in revised form 18 June 2018; accepted 25 June 2018 DOI https://doi.org/10.21595/chs.2018.19954

Check for updates

Copyright (C) 2018 Kamyar M. Hedayat, et al. This is an open access article distributed under the Creative Commons Attribution License, which permits unrestricted use, distribution, and reproduction in any medium, provided the original work is properly cited.

\begin{abstract}
Background: Chronic heart failure (CHF) is an inflammatory disorder in which cortisol plays an important role. Despite this, cortisol is not routinely quantitatively measured for a number of reasons. It is considered non-specific. Accuracy and validity remain in question. It is not considered convenient or cost effective. Finally, tissue level effects of cortisol do not correlate linearly to quantitative levels. If the functional, tissue level effectiveness of cortisol could be modeled, its evaluation in CHF patient may become relevant. Endobiogeny is a global systems theory that claims to be able to model complex physiology through biomarkers, offering contextrich interpretations of data for meaningful clinical applicability. Cortisol is known to alter circulating levels of elements from a complete blood count (CBC). By relating these biomarkers in a qualitative fashion, the theory of Endobiogeny posits that these elements can be contextualized to reflect the tissue level activity of cortisol, referred to as the cortisol index (CI). The algorithm derived from the theory is called the Biology of Functions (BoF). Aim: The aim of this study was to determine if the cortisol index is accurate in reflecting a greater expression of cortisol activity in ambulatory $\mathrm{CHF}$ patients versus controls subjects. Methods: A retrospective observational case control study was performed in 93 patients with New York Heart Association class II-III heart failure patients and 104 individuals with no cardiovascular pathology as a control group. Results from a $\mathrm{CBC}$ were entered into BOF modeling software, from which the cortisol index is derived. Results: The Cortisol index (3-7) was significantly elevated in CHF vs. control patients (12.8 \pm 0.91 vs. $8.48 \pm 0.74, p<001)$, as were individual CBC elements used to form the index. Conclusions: The cortisol index, derived from the theory of Endobiogeny showed results consistent with CHF pathophysiology. The cortisol index was able to model the effective tissue level activity of cortisol in CHF patients using only a CBC, without measuring serum cortisol. Future studies should compare the cortisol index to standard inflammatory markers in CHF patients to further correlate the validity of the index to other known effects of cortisol.
\end{abstract}

Keywords: systems biology, biological markers/analysis, hormones, metabolism, heart failure, humans.

\section{Introduction}

Cortisol is a key hormone involved in the adaptation response. Its role in the pathophysiology of chronic heart failure (CHF) is well characterized [1-3]. Elevated serum cortisol is correlated 
with morbidity and mortality in CHF. Despite this, it is not routinely evaluated for four reasons: clinical utility, convenience, convention and accuracy. If these issues can be addressed, cortisol assessment may prove to be of value in CHF patients.

Perceived lack of clinical utility of cortisol evaluation arises from three considerations: emphasis on downstream biomarkers, lack of specificity and absence of pharmacologic treatment. Over the last few decades, research has focused on increasingly downstream markers of CHF pathophysiology, e.g. interleukin-6 and matrix metalloproteinase (MMP) [4]. From this perspective, cortisol is seen as too far upstream to be of value. Early research in CHF was oriented towards a systems approach $[2,3]$. Since CHF is a multisystem dysfunction, returning to this approach of studying upstream mediators may be beneficial.

Cortisol lacks specificity in CHF. In contrast, brain-derived natriuretic peptide (BNP) is tissue-specific, correlating to severity of disease and mortality [4]. However, CHF influences and is influenced by vascular, renal, pulmonary, endocrine and autonomic dysfunction, not just myocardial dysfunction. Since cortisol affects all these factors in CHF patients, $[1,5]$ evaluating the constitutive role of cortisol may be more valuable in managing patients than only investigating markers of myocardial dysfunction.

Finally, despite extensive research, no commercially available pharmacologic treatments are available to reduce cortisol [6]. However, the same can be said for IL-6, MMP and BNP. Furthermore, unlike downstream markers, non-pharmacologic interventions have been shown to lower cortisol in various clinical conditions, including cardiovascular disease [7-9].

Convenience is the second reason cortisol is not routinely evaluated in CHF. Serum cortisol tests are neither routine nor rapid. Furthermore, the validity of morning vs. random cortisol blood draws has not been satisfactorily established [10]. Convention is the third reason. Hormone assessment is considered the domain of endocrinologists, not cardiologists. However, cardiologists manage cardiac disease through regulation of neuroendocrine factors viz. inhibitors of adrenaline, aldosterone, angiotensin, etc. Longitudinal evaluation of cortisol would be within the scope of clinical cardiology if it were proven to have diagnostic or predictive value in CHF.

Accuracy and validity are the third reason cortisol is not routinely evaluated. Discoveries in molecular endocrinology have raised questions regarding the value of quantitative serum cortisol measurement. Cortisol's actions can be genomic or non-genomic [11]. Many non-genomic actions of cortisol are permissive, [12] viz. sequestration of inflammatory immune elements, and improved cardiac function [11, 13, 14]. Genomic effects involve several mechanisms for which genetic polymorphisms and epigenetic influences can alter efficiency and activity of cortisol [5, 11]. Genomic effects are primarily adaptive, acting via NF- $\kappa B$ up-regulation [11]. These adaptive actions of cortisol are the ones association with severity of CHF. In summary, a quantitative assessment of serum cortisol may not be a reliable indicator of its downstream functional effects on metabolism.

The theory of endobiogeny is a global systems approach to integrative physiology [15]. As such, it favors evaluation of upstream causes of pathophysiology - such as cortisol - over downstream mechanisms of disease such as IL-6, MMP and BNP. From the theory of endobiogeny a physiologic modeling system has been derived called the biology of functions (BoF). Biomarkers are viewed as downstream products of cellular metabolism and thus indirect markers of upstream regulation of cell and tissue metabolism [16]. The applicability of this global systems approach in CHF patients was recently demonstrated with respect to the neutrophil-to-lymphocyte ratio, referred to as the Genito-thyroid index in Endobiogenic terminology [17].

It is claimed by proponents of the theory Endobiogeny that the functional activity of cortisol can be modeled without measuring cortisol in any body fluid [16]. An index has been developed called the Cortisol index (CI). The proprietary formula is derived from published studies on the action of cortisol on bone marrow and its cellular elements in circulation. Thus, the theory takes the effects of cortisol on a single type of tissue - bone marrow - and extrapolates this to consider the global functional effects of cortisol on all tissues.

Cortisol increases production and circulating levels of leukocytes in general, and neutrophils 
in particular. It diminishes circulating levels of lymphocytes and eosinophils through sequestration and apoptosis. We have evaluated these studies in detail elsewhere [16]. The cortisol index formula then incorporates the bone marrow products that are increased in the numerator, and those that are diminished by cortisol in the denominator. In the context of heart disease, these changes in the CBC have already been characterized, $[17,18]$ but not evaluated simultaneously according to a global systems perspective until now.

We hypothesized that the modeling system of endobiogeny would demonstrate elevated values of the cortisol in ambulatory CHF patients versus controls.

\section{Methods}

\subsection{Study participants}

One hundred and four control subjects were recruited with no history of cardiovascular pathology, and ninety-three subjects with New York Heart Association (NYHA) classes II through III heart failure were recruited from the San Diego Veterans Affairs Medical Center Cardiology Clinic and the University of California, San Diego (UCSD) Medical Center Advanced Heart Failure Program for this retrospective observational case-controlled study. They were part of a larger study in subjects with heart failure in which the effects of depression on cellular adhesion and inflammation was being investigated. We included the non-CHF control sample from the local community via advertisements (e.g., newspapers, flyers, brochures, and websites) and word of mouth referrals.

The first inclusion criteria were age between 30 and 85 years. Additional inclusion criteria for non-control subjects were optimal standard management of heart failure for three months or longer. As part of the patient's routine medical evaluation, left ventricular ejection fraction (LVEF) was assessed by echocardiography. Exclusion criteria included recent history of myocardial infarction, stroke or significant cerebral neurological impairment within the past month. Other criteria included presence of severe chronic obstructive pulmonary disease, psychiatric illness (other than depression), or anxiety. Subjects were asked to avoid aspirin for 1 day before testing.

The investigation conformed to the principles outlined in the Declaration of Helsinki. The University of California, San Diego Institutional Review Board, approved the study. All subjects gave informed written consent.

\subsection{Biochemical analyses}

For complete blood count with differential and platelets, blood was drawn into ethylenediaminetetraacetic acid (EDTA)-coated vacutainer tubes (BD Biosciences, San Jose, California). The Clinical Laboratory at the UCSD Medical Center resulted all blood samples.

\subsection{Value of indices}

The normal value of the cortisol index for adults is 3-7. The indices of the biology of functions (BoF) are ratios or relative physiologic activity, therefore they contain no units of value. They were derived through internal research on healthy vs. non-healthy populations of Western European origin.

The BoF models both structure vs. function values. The Cortisol index contains both structure and function values. Structure values evaluate basil function. Function values model adaptive activity. In this population of CHF patients, the function value of cortisol was used, since we theorize CHF to be a functional adaptation disorder of the myocardium. 


\subsection{Statistical analyses}

Using the Kolmogorov-Smirnov test, normal distribution of data was verified prior to statistical analyses for both groups. Data for cortisol and eosinophils underwent log transformation. We calculated mean arterial pressure (MAP) and body mass index (BMI) using standard formulas. Data are presented as mean \pm standard distribution. Results were considered statistically significant at the $p \leq 0.05$ level. Using Pearson correlation, the relationship among variables was tested and by analysis of variance the groups were compared. All calculations were performed using SPSS Inc. (version 24.0) software package (SPSS, Chicago, Illinois).

In Table 1, sociodemographic and medical characteristics of the study participants are presented. Heart failure patients were older $(p<0.01)$ and with greater BMI $(p<0.01)$ than non-HF subjects. Heart failure patients also had lower mean blood pressure $(p<0.01)$.

Table 1. Sociodemographic and medical characteristics of the study subjects

\begin{tabular}{|c|c|c|}
\hline Mean (SD) & Heart failure & Non-heart failure \\
\hline$N$ & 93 & 104 \\
\hline Age (years) * & $55.9(12)$ & $49.2(14)$ \\
\hline Gender (\#Women / \#Men) & $15 / 78$ & $12 / 92$ \\
\hline Body Mass Index $\left[\mathrm{kg} / \mathrm{m}^{2}\right]^{*}$ & $31.9(8.2)$ & $28.8(6.4)$ \\
\hline Mean arterial pressure $[\mathrm{mm} \mathrm{Hg}]$ ** & $80.0(9.5)$ & $90.5(10.1)$ \\
\hline LVEF (\%) & $32.1 \%$ & - \\
\hline \multicolumn{3}{|c|}{ Medications } \\
\hline ACE inhibitors & $73 \%$ & $0 \%$ \\
\hline Beta blockers & $95 \%$ & $0 \%$ \\
\hline Calcium channel blockers & $5 \%$ & $0 \%$ \\
\hline Statin & $56 \%$ & $0 \%$ \\
\hline Aspirin & $54 \%$ & $7 \%$ \\
\hline Diuretics & $90 \%$ & $0 \%$ \\
\hline Anti-arrhythmics & $9 \%$ & $0 \%$ \\
\hline Digoxin & $61 \%$ & $0 \%$ \\
\hline${ }^{*} p<0.01,{ }^{* *} p<0.001$ & & \\
\hline
\end{tabular}

The Cortisol index was significantly different between HF and control (12.8 \pm 0.91 vs. $8.48 \pm 0.74, p<001)$. Evaluation of the individual components of the CBC with differential and the genito-thyroid index (neutrophil/lymphocytes) demonstrated a significant correlation for all components except eosinophils (Table 2).

Table 2. ANOVA of heart failure vs. control

\begin{tabular}{|c|c|c|c|c|c|c|c|}
\hline Variable & Normal range & HF & Control & Combined & F value & DF & Partial Eta \\
\hline Cortisol index* & $3-7$ & $12.8 \pm 8.5$ & $8.48 \pm 7.4$ & $10.5 \pm 8.2$ & 15.87 & 1.188 & 0.078 \\
\hline WBC $^{*}$ & $5000-8500 / \mu \mathrm{L}$ & $6.952 \pm 1.725$ & $5.791 \pm 1.456$ & $6.339 \pm 1.687$ & 26.18 & 1.195 & 0.118 \\
\hline Neutrophils* & $45-55 \%$ & $62 \pm 10$ & $58 \pm 8$ & $60 \pm 9$ & 12.94 & 1.195 & 0.062 \\
\hline Lymphocytes* & $35-45 \%$ & $25 \pm 9$ & $30 \pm 7$ & $28 \pm 8$ & 18.92 & 1.195 & 0.088 \\
\hline Eosinophils** & $1-5 \%$ & $3.57 \pm 3.6$ & $3.32 \pm 2.4$ & $3.44 \pm 3.1$ & 0.427 & 1.195 & 0.002 \\
\hline
\end{tabular}

There was a significant positive association between the cortisol index total WBC count and percent neutrophils on differential, and significant negative association with percent lymphocytes and eosinophils on differential (Table 3).

The relationship between various elements of the CBC used to compose the cortisol index was compared for the all patients (HF and control). There was a significant positive correlation between total WBC count and the relative percentage of neutrophils and lymphocytes, but not too percent eosinophils (Table 4). There was a significant negative correlation between percent neutrophils and both eosinophils and lymphocytes (Table 4). 
Table 3. Pearson correlation per group

\begin{tabular}{|c|c|c|c|c|}
\hline Cortisol index & WBC & Neutrophils & Lymphocytes & Eosinophils \\
\hline HF $(n=89)$ & $0.420^{*}$ & $0.678^{*}$ & $-0.510^{*}$ & $-0.708^{*}$ \\
\hline Control $(n=101)$ & $0.370^{*}$ & $0.536^{*}$ & $-0.424^{*}$ & $-0.735^{*}$ \\
\hline Combined $(n=190)$ & $0.450^{*}$ & $0.633^{*}$ & $-0.520^{*}$ & $-0.676^{*}$ \\
\hline${ }^{*} p<0.001$; WBC: white blood cell \\
\hline
\end{tabular}

Table 4. Pearson correlation of the $\mathrm{CBC}$ with differential

\begin{tabular}{|c|c|c|c|c|}
\hline & WBC & Neutrophils & Lymphocytes & Eosinophils \\
\hline WBC & 1 & $0.463^{*}$ & $-0.420^{*}$ & $-0.119^{* *}$ \\
\hline Neutrophils & $0.463^{*}$ & 1 & $-0.906^{*}$ & $-0.281^{*}$ \\
\hline${ }^{*} p<0.001,{ }^{* *} p>0.05$ \\
\hline
\end{tabular}

\section{Discussion}

In this retrospective study, we evaluated the validity of a formula that models the effective tissue level activity of cortisol in patients with CHF. We found the mean value of the Cortisol index (CI) higher in CHF patients compared to controls. This is in accordance with over 50 years of research in this patient population [1-3]. In general, patients with cardiovascular disease are more sensitive to glucocorticoids than control subjects.

At this time there is consensus neither on which body fluid is the most appropriate source of measurement nor how accurate or valid quantitative measurement of cortisol may be. Stricto senso, it only evaluates metabolic output from the adrenal cortex. Thus, it is suggestive of potential tissue activity but not its actual achieved effects, [10] which depend on a myriad of genetic and epigenetic factors $[5,15,16]$. Fasting plasma cortisol may not be a reliable indicator of tissue-level cortisol activity [5]. Salivary or urinary cortisol may be more representative of this as a posteriori measurement of cortisol movement through tissues, but they are not routinely evaluated [10]. Some studies have found salivary - not serum - cortisol predictive of all-cause mortality in general, once again raising questions about the value of serum cortisol evaluation [19].

The advantage of the cortisol index according to the theory of Endobiogeny is that it is easily, rapidly and inexpensively calculated from a completed blood count with differential and platelets. This test is commonly performed in ambulatory CHF patients. Because it models net global functional activity, it is not dependent on circadian rhythms and can be drawn at any time. It can be repeatedly performed even within hours of prior samples to evaluate the evolution of cortisol activity in acute and chronic settings [16, 17].

In this study, elements of a $\mathrm{CBC}$ affected by cortisol were found to have statistical significance with the cortisol index. However, no single element of a CBC alone could be used to evaluate cortisol activity. Furthermore, when evaluating the relationship between individual components of the formula, both the qualitative relationship of percent leukocytes (neutrophils, leukocytes and eosinophils) and the total, quantitative assessment of white blood cells was required to properly account for all the influences on the formula. We find that this supports the Endobiogenic theory that it is in both quantitative and qualitative assessments that physiologic activity can be best characterized.

There are limitations to this study. The index selects a single tissue: bone marrow as representative of all tissues in which cortisol has an effect. This assumption has not been validated though we are not sure how it could be because of the lack of biomarkers as relatively easily obtained as those from the bone marrow. Furthermore, the crucial and foundational role of marrow and the elements immunity, oxygenation and thrombosis that it produces makes it the essential and constitutional tissue.

The study was retrospective in nature and the prognostic role of cortisol or the cortisol index could not be determined. The cortisol index was not compared to quantitative measurements of cortisol by serum, saliva or urine. However, as we have discussed above, it is not clear how valid 
these measurements would be as a "gold standard" of cortisol activity. Future studies should be prospective in nature to evaluate the prognostic value of the cortisol index.

As a stand-alone index, the cortisol index may prove to be of value in evaluating one aspect of CHF physiology. The biology of functions incorporates the cortisol index into meta-indexes that purport to model complex states and events affected by cortisol such as inflammation and risk of arterial wall damage and thrombolysis [16]. Future studies will need to validate these claims.

\section{Conclusions}

This study demonstrated for the first time that tissue-level actions of cortisol can be modeled based on the know effects of cortisol on a $\mathrm{CBC}$ with differential according to a global systems approach based on the theory of Endobiogeny. A positive correlation was shown between mean cortisol in ambulatory patients with stages 2 or 3 CHF versus control subjects. The cortisol index appears to meet all the criteria hypothesized at the outset for the ideal marker of cortisol activity: rapid, inexpensive and quantitative assessment of the qualitative effects of cortisol on tissue.

\section{References}

[1] Anand I. S., Ferrari R., Kalra G. S., Wahi P. L., Poole Wilson P.-A., Harris P. C. Edema of cardiac origin. Studies of body water and sodium, renal function, hemodynamic indexes, and plasma hormones in untreated congestive cardiac failure. Circulation, Vol. 80, Issue 2, 1989, p. 299-305.

[2] Connolly C. K., Wills M. R. Plasma cortisol levels in heart failure. British Medical Journal, Vol. 2, Issue 5543, 1967, p. 25-27.

[3] Knapp M. S., Keane P. M., Wright J. G. Circadian rhythm of plasma 11-hydroxycorticosteroids in depressive illness, congestive heart failure, and Cushing's syndrome. British Medical Journal, Vol. 2, Issue 5543, 1967, p. 27-30.

[4] Braunwald E. Biomarkers in heart failure. The New England Journal of Medicine, Vol. 358, Issue 20, 2008, p. 2148-2159.

[5] Walker B. R. Glucocorticoids and cardiovascular disease. European journal of endocrinology. European Federation of Endocrine Societies, Vol. 157, Issue 5, 2007, p. 545-559.

[6] Boyle C. D. Recent advances in the discovery of 11beta-HSD1 inhibitors. Current Opinion in Drug Discovery and Development, Vol. 11, Issue 4, 2008, p. 495-511.

[7] Chellew K., Evans P., Fornes Vives J., Perez G., Garcia Banda G. The effect of progressive muscle relaxation on daily cortisol secretion. Stress, Vol. 18, Issue 5, 2015, p. 538-544.

[8] Mcgrady A. V., Yonker R., Tan S. Y., Fine T. H., Woerner M. The effect of biofeedback-assisted relaxation training on blood pressure and selected biochemical parameters in patients with essential hypertension. Biofeedback and Self-Regulation, Vol. 6, Issue 3, 1981, p. 343-353.

[9] Atsumi T., Tonosaki K. Smelling lavender and rosemary increases free radical scavenging activity and decreases cortisol level in saliva. Psychiatry Research, Vol. 150, Issue 1, 2007, p. 89-96.

[10] Vogelzangs N., Beekman A. T., Milaneschi Y., Bandinelli S., Ferrucci L., Penninx B. W. Urinary cortisol and six-year risk of all-cause and cardiovascular mortality. The Journal of Clinical Endocrinology and Metabolism, Vol. 95, Issue 11, 2010, p. 4959-4964.

[11] Kadmiel M., Cidlowski J. A. Glucocorticoid receptor signaling in health and disease. Trends in Pharmacological Sciences, Vol. 34, Issue 9, 2013, p. 518-530.

[12] Garrod O. The pharmacology of cortisone, cortisol (hydrocortisone) and their new analogues. Postgraduate Medical Journal, Vol. 392, Issue 34, 1958, p. 300-304.

[13] Oakley R. H., Cidlowski J. A. The biology of the glucocorticoid receptor: new signaling mechanisms in health and disease. The Journal of Allergy and Clinical Immunology, Vol. 132, Issue 5, 2013, p. 1033-1044.

[14] Groeneweg F. L., Karst H., De Kloet E. R., Joels M. Rapid non-genomic effects of corticosteroids and their role in the central stress response. The Journal of Endocrinology, Vol. 209, Issue 2, 2011, p. 153-167.

[15] Lapraz J. C., Hedayat K. M. Endobiogeny: a global approach to systems biology (part 1 of 2). Global Advances in Health and Medicine: Improving Healthcare Outcomes Worldwide, Vol. 2, Issue 1, 2013, p. 64-78. 
[16] Lapraz J. C., Hedayat K. M., Pauly P. Endobiogeny: a global approach to systems biology (part 2 of 2). Global Advances in Health and Medicine: Improving Healthcare Outcomes Worldwide, Vol. 2, Issue 2, 2013, p. 32-44.

[17] Hedayat K., Schuff B., Lapraz J., Barsotti T., Golshan S. Genito-Thyroid index: a global systems approach to the neutrophil-to-lymphocyte ratio according to the theory of endobiogeny applied to ambulatory patients with chronic heart failure. Journal of Cardiology and Clinical Research, Vol. 5, Issue 1, 2017, p. 1091.

[18] Cikrikcioglu M. A., Soysal P., Dikerdem D., et al. Absolute blood eosinophil count and 1-year mortality risk following hospitalization with acute heart failure. European Journal of Emergency Medicine: Official Journal of the European Society for Emergency Medicine, Vol. 19, Issue 4, 2012, p. 257-263.

[19] Schoorlemmer R. M., Peeters G. M., Van Schoor N. M., Lips P. Relationships between cortisol level, mortality and chronic diseases in older persons. Clinical Endocrinology, Vol. 71, Issue 6, 2009, p. $779-786$. 